\title{
Brain-Computer Interfacing for Wheelchair Control by Detecting Voluntary Eye Blinks
}

\author{
William C Francis', C. Umayal ${ }^{2}$, G. Kanimozhi ${ }^{3}$ \\ 1,2,3 School of Electrical Engineering, VIT University Chennai Campus, Melakottaiyur, Chennai-600127, India
}

\begin{tabular}{l}
\hline \hline Article Info \\
\hline Article history: \\
Received Oct 6, 2020 \\
Revised May 11, 2021 \\
Accepted May 27, 2021 \\
\hline
\end{tabular}

\section{Keyword:}

Electroencephalography

Brain Computer Interface

Brainwaves

Wheelchair control

Non-invasive EEG

EEG electrodes

\begin{abstract}
The human brain is considered one of the most powerful quantum computers and combining the human brain with technology can even outperform artificial intelligence. Using a Brain-Computer Interface (BCI) system, the brain signals can be analyzed and programmed for specific tasks. This research work employs BCI technology for a medical application that gives the unfortunate paralyzed individuals the capability to interact with their surroundings solely using voluntary eye blinks. This research contributes to the existing technology to be more feasible by introducing a modular design with three physically separated components: headwear, a computer, and a wheelchair. As the signal-to-noise ratio (SNR) of the existing systems is too high to separate the eye blink artifacts from the regular EEG signal, a precise ThinkGear module is used which acquired the raw EEG signal through a single dry electrode. An embedded Bluetooth module acquires and transfers the signals wirelessly to a computer. A MATLAB program captures voluntary eye blink artifacts from the brain waves and commands the movement of a miniature wheelchair via Bluetooth. To distinguish voluntary eye blinks from involuntary eye blinks, blink strength thresholds are determined. A Graphical User Interface (GUI) designed in MATLAB displays the EEG waves in realtime and enables the user to determine the movements of the wheelchair which is specially designed to take commands from the GUI. The findings from the testing phase unveil the advantages of a modular design and the efficacy of using eye blink artifacts as the control element for brain-controlled wheelchairs. The wheelchair attained a command detection and execution accuracy of $96.4 \%$ which is an improvement from the existing systems. The work presented here gives a basic understanding of the functionality of a BCI system and provides eye blink-controlled navigation of a wheelchair for patients suffering from severe paralysis.
\end{abstract}

Copyright $@ 2018$ Institute of Advanced Engineering and Science. All rights reserved.

\footnotetext{
Corresponding Author:

Dr.Kanimozhi.G,

School of Electrical Engineering, VIT University,

Melakottaiyur, Chennai-600127.

Email: kanimozhi.g@vit.ac.in
}

\section{INTRODUCTION}

Over hundreds of years, scientists have spent a lifetime studying the clockwork inside a human brain. The history of neurosciences dates back to ancient pharaonic civilization but they lacked the resources to conduct studies on the human brain. They even considered the heart to be the origin of human thoughts, as discussed in [1]. With the rise of modern technology and advancements in the field of biomedical engineering, this research has given way to a new term known as Brain-Computer Interfacing.

A human brain comprises of billions of neurons and collectively generates about 12-25 watts of electricity, which is enough to power an LED light bulb. The BCI technology utilizes this electricity that is 
being generated in the brain to monitor and analyze brain activity. It converts the electrophysiological signals from the Central Nervous System (CNS) into instructions that the computer understands. Brain signal goes directly from the brain to the computer instead of traveling through the nervous system to the fingertips to the mouse, keyboard, or a peripheral input device of the computer. It has been estimated that by the year 2050, BCI would make it possible for humans to control daily objects with their minds [2]. BCI is a powerful tool that bridges the gap between the human brain and the computer. It is a booming field of research with a wide array of applications ranging from medical to gaming and entertainment. It is often used in biomedical applications to observe the brain activity of a person or to deliver his/her commands to a computer.

Brain-controlled wheelchair for physically disabled people is a topic that is deeply researched upon since it provides locomotive abilities to the disabled without moving their limbs. BCI is required to acquire the EEG signals from the human brain and to convert it into a movement instruction for the wheelchair. However, the electrical signals produced in the brain are considerably minute in voltage and current magnitude. Therefore, a BCI system for wheelchair control comprises the following components: Signal Acquisition, Signal Preprocessing, Feature Extraction, Classification, and Wheelchair Motor Control.

Signal acquisition is the measurement of electrophysiological signals from the brain using electrodes that are placed on the scalp of the head [3]. Signal pre-processing deals with filtering the noise in the signal that is acquired by electrodes and amplifying it to a magnitude perceivable by the computer. The next step, feature extraction, extracts certain features from the signal which corresponds to a particular brain activity or a command for action. In this research work, the feature that needs to be extracted from the EEG signal is called Eye blink artifact. Each time the subject blinks their eye, it is extracted [4] and classified as a command for movement of the wheelchair in a specific direction. This completes the classification step. Finally, the movement command from the classification process [5] is carried out by the motors of the wheelchair, thereby mapping the EEG signals to corresponding wheelchair movements. Figure. 1 illustrates this process flow in a BCI system.

Previous researches have explored various methods of signal acquisition, signal preprocessing, feature extraction, and classification for BCI systems in wheelchair control applications. EEG is often the mode of signal acquisition when the targeted users are severely paralyzed individuals. However, one drawback of EEG systems is the need for specialized hardware. Many BCI systems that target less severely paralyzed individuals employ intrusive gaze-controlled [6] or eye blink-controlled communication [7]. Such systems use either of the two hardware devices: electro-oculographs (EOG) [8] or infrared (IR) devices [9]. The EOG measures the corneoretinal potential using skin electrodes near the lateral and medial canthus. This potential changes with eye movement and can be used for tracking eye movement. An IR device, on the other hand, requires an infrared light source and a camera. The IR beam from the source reflects off the retina of the eye and can be captured by the camera, enabling detection and tracking of the eyes. These patterns of eyeball movements or voluntary eye blinks are associated with movement commands to a wheelchair. A recent study [10] on electrooculography for Human-Machine Interface (HMI) showcases the effectiveness of EOG-controlled wheelchair in rehabilitating individuals suffering from motor degenerative diseases. This particular EOG-based system attained a command detection and execution accuracy of $93.89 \%$ without employing a Graphical User Interface. A hybrid of EEG and EOG has also been successfully employed for wheelchair navigation [11]. By combining EEG with EOG, the BCI system could utilize eye blinking, motor imagery, and P300 potentials to implement a greater number of control commands and a greater information transfer rate.

Many people suffer from paralysis as a result of damage in their nervous system, especially the spinal cord, or due to Amyotrophic Lateral Sclerosis (ALS). With the loss of motor functions, these individuals are forced to be dependent on others for fulfilling their daily needs and care $[4,12,13]$. Unfortunately, this leads to a lower quality of life for them. Although there exist human-computer interfaced systems that allow the paralyzed to control their wheelchair by speech and retina-controlled computing, they do not address the needs of severely paralyzed patients who cannot speak or move their retina. Previous researches like [14] have attempted to solve this issue using facial recognition to detect eye blinks. A simple vision-based eye blink monitoring system uses a single camera to take successive images of the face. The captured images are classified as ,eyes open ${ }^{\text {ee }}$ or ,eyes closed ${ }^{\text {ee }}$ by using an image processing technique. Usually, a machine learning or a deep learning algorithm takes care of the classification task. An eye blink is registered if the successful images are classified differently. However, this technique requires a camera to be focused on the user at all times and it is computationally expensive. This problem can be tackled by taking real-time EEG signals from the patients ${ }^{\text {ee }}$ scalp and decoding the brain activity to control the wheelchair

\section{PRINCIPLES OF EEG}

Electroencephalography is one of the physiological tools required to measure the electrical activity generated by the brain, and this is done by placing electrodes on the scalp of the head. It is the only noninvasive test to acquire brain patterns, and this helps in medical diagnoses ranging from headaches and epilepsy

IJEEI, Vol. 9, No. 2, June 2021: 521 - 537 
to major conditions such as brain tumors and dementia. The simultaneous firing of many neurons in the brain creates very small electric signals and this pattern of waves are picked up by the electrodes placed on the

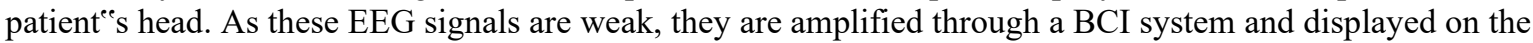
computer screen. The waves recorded provides real-time electrical activity [15] and mainly interprets which cerebral cortex is being used to gather information at that time.

\subsection{EEG Wave Groups}

The brain waves have frequency ranges that differ with mental performance, especially on the thought processes and sensations. Each brain wave differs depending on the amplitude and frequency [16] as shown in Figure. 3.

i. Gamma waves: Gamma waves constitute the parts of the brain wave between $32 \mathrm{~Hz}$ and $100 \mathrm{~Hz}$. The high frequency relates to a peak mental state where multiple information can be processed and allows stronger perception [16].

ii. Beta waves: Beta waves coincides with complex thoughts and engages in analytical problem-solving abilities. Human adults spend most of their awake hours in this state and hence it is split into 3 bands namely,

a. Beta1 (13-15 Hz) - Thinking, fast idle

b. Beta2 (15-22 Hz) - Actively thinking and Figuring out

c. Beta3 $(22-32 \mathrm{~Hz})-$ Complex thoughts, anxiety, new experiences

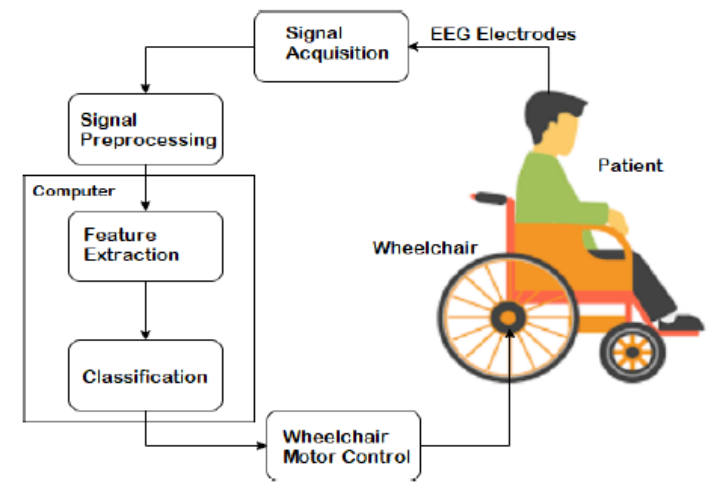

Figure. 1. Block diagram of BCI system for wheelchair control

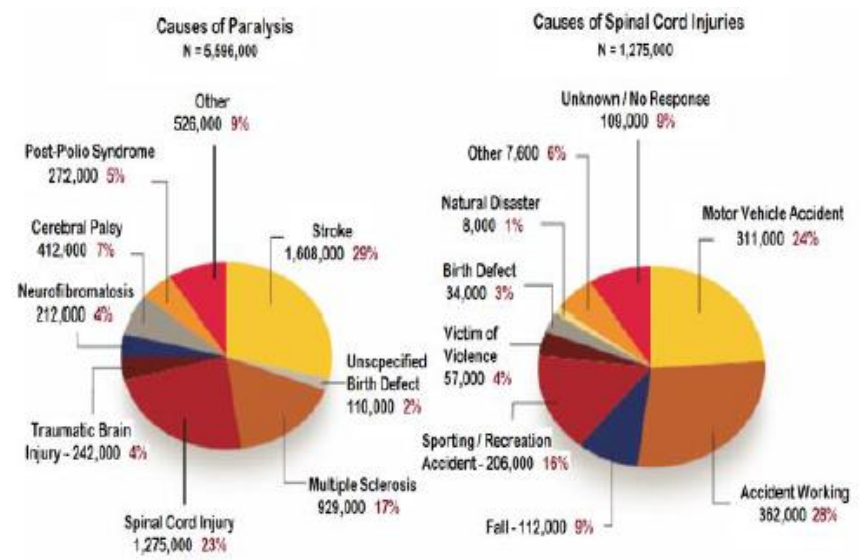

Figure. 2. Statistical analysis of the causes of spinal cord injuries and paralysis

iii. Alpha waves: This group dominates the meditative or resting states and aids in calmness [16]. When eyes are blinked, the wave group $(8-13 \mathrm{~Hz})$ is affected. This spontaneous change in electric potential is detected and further used as a control element in this research work.

iv. Theta waves: The curves of these waves $(4-8 \mathrm{~Hz})$ indicate deep relaxation and associate in monitoring deep meditation as we focus on our thoughts within. This state proves the best form to learn and improve memory. 
v. Delta waves: The loudest and slowest brainwave frequency $(0.5-4 \mathrm{~Hz})$ indicates the deepest meditation and dreamless sleep. Even healing powers are stimulated in this state and hence crucial for each individual to achieve during their sleep.

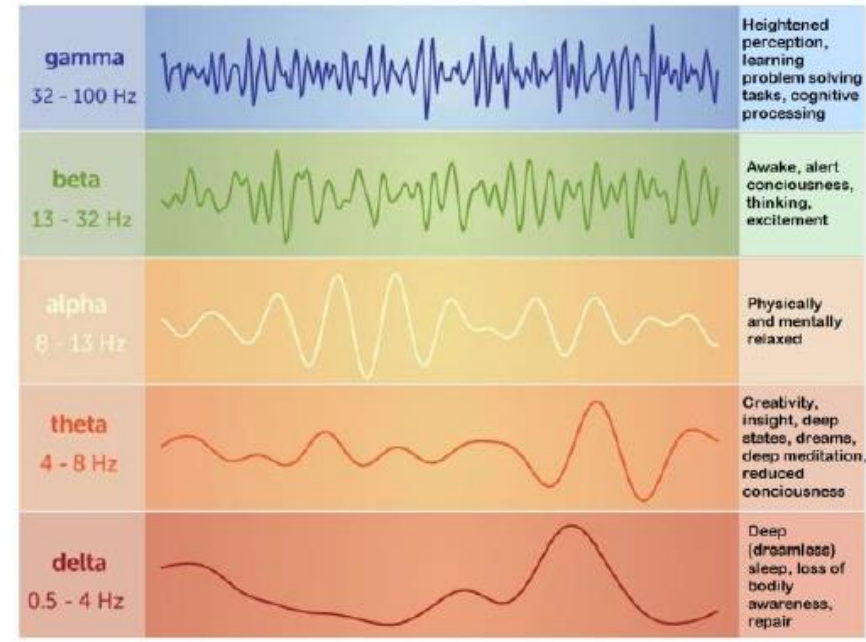

Figure. 3. Classification of brain waves based on frequency
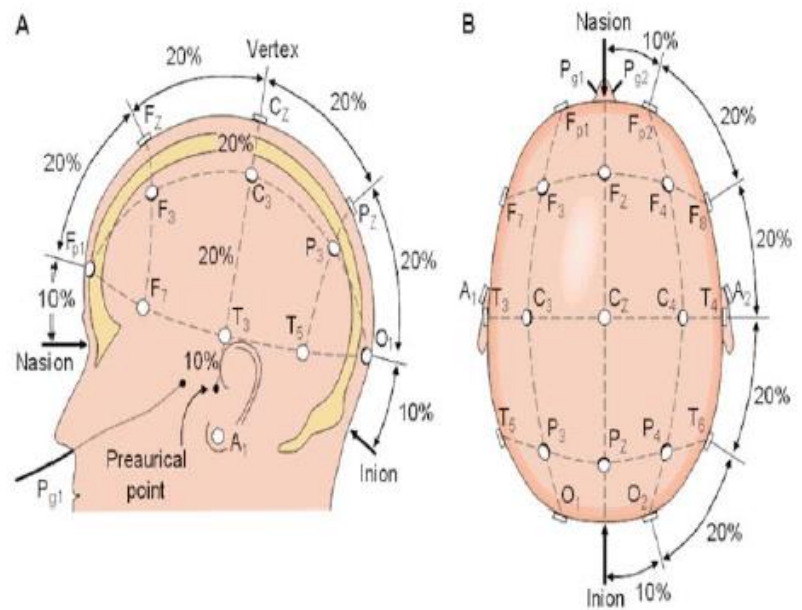

Figure. 4. Illustration of the 10-20 EEG electrode placement system

\subsection{The 10-20 System of Electrode Placement}

The electrodes that need to be placed on the scalp of the head are followed by an internationally recognized standard known as the 10-20 system [17]. This system is strictly in relation to the location of the electrodes and the cerebral cortex. Mainly, it ensures that the inter-electrode spacing is equal as shown in Figure. 4. The ,10 $10^{\text {ee }}$ and the ,20" signify the distance between adjacent electrodes. It corresponds to $10 \%$ or $20 \%$ covering the entire head either from front to back or left to right. Frontal (F), Central(c), Parietal (P), Temporal (T)and Occipital regions $(\mathrm{O})$ are EEG electrodes. Each placement is numbered according to the two hemispheres. The right hemisphere is for even numbers from 2 to 8 and the left hemisphere is for the odd numbers from 1 to 9 .

\subsection{Eye Blink Artifact}

The brain signal obtained by the EEG electrodes comprises all kinds of neural activity in and around the brain. Every thought, every motor command, and every feedback from the neurons in the body adds to this electrical activity. This means that every time we close and open our eyes, there is a disturbance in the electrical potentials on the scalp. This is one of the most prominent changes in the EEG signal that creates a noticeable trough in the brain wave as shown in Figure 5. This event in the brain wave when we blink our eyes is known as an eye blink artifact. 


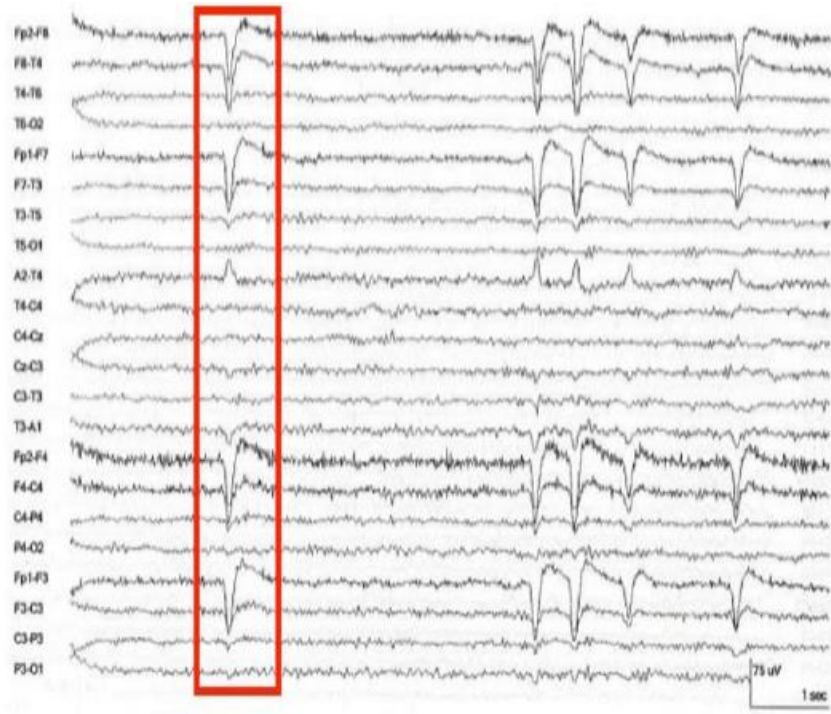

Figure 5. Eye blink artifacts in EEG waveform

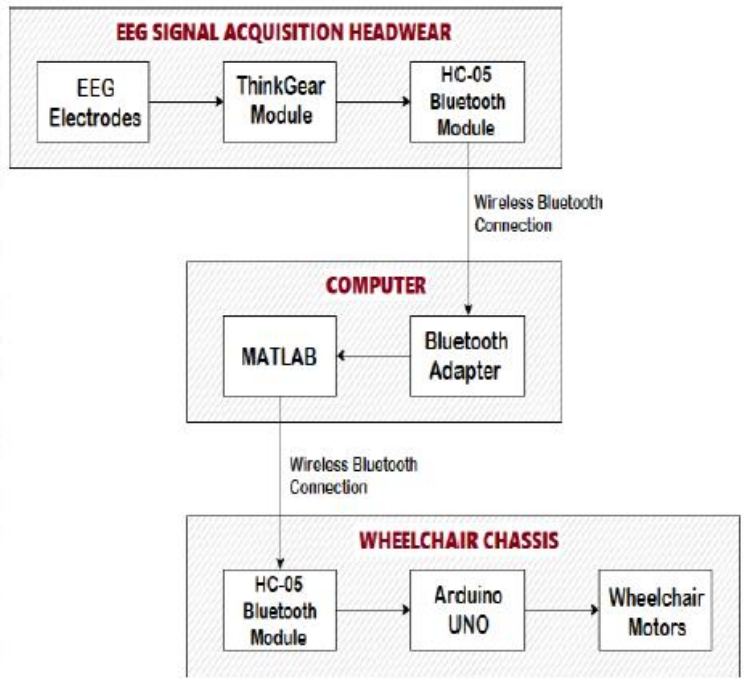

Figure 6. Block diagram of the brain-controlled wheelchair

Eye blink artifacts are often considered as a contamination in the EEG signals and are removed by using Artificial Neural Networks (ANN). However, we looked at the bright side of eye blink artifacts and put them to use. They are used as an input [4] from the paralyzed patients to control their wheelchair. The few advantages of using eye blink artifacts as the control element are as follows:

i. Eye blink artifacts are easily noticeable in the brain waves because eye blinks cause large disturbances [18] in the electric potentials.

ii. Eye blink artifacts can be easily detected without the need for complex machine learning algorithms like in [19].

iii. Even the most severely paralyzed patients can open and close their eyes. Thus, making it suitable for the majority of the paralyzed patients.

iv. Eye blink artifacts can be obtained from the forehead alone, making EEG electrode placement easier. Thick hair often results in weak signals and high noise. Placing the electrodes on the forehead eliminates this problem.

\section{DESIGN OF BRAIN CONTROLLED WHEELCHAIR}

\subsection{Design Approach}

The design of the Brain Controlled Wheelchair is physically separated into three components: an EEG signal acquisition system on the head $[13,20]$, a computer that makes wheelchair navigation simple, a wheelchair chassis that can take commands from the computer and move accordingly. These components are

Brain-Computer Interfacing for Wheelchair Control by Detecting Voluntary...(William C Francis et al) 
interconnected via Bluetooth as shown in Figure 6. From the previous researches conducted on this topic, we learned that even after passing the EEG signal through a low noise amplifier and several filter circuits, there are enigmatic disturbances in the EEG wave whose sources are unknown. Although a human can differentiate these spikes from eye blink artifacts, it would be near impossible for a computer, without using machine learning. ThinkGear ASIC Module (TGAM) is a popular EEG technology that senses faint signals from the brain, amplifies, and filters it, and converts them to digital signals [21]. This module is integrated with dry silver electrodes and a Bluetooth module which makes up the components of the headwear. The electrodes pick up differences in electric potential at FP1 [17] and the TGAM pre-processes this signal and the Bluetooth module transmits this signal to a computer.

The computer acts as the brain of this work, taking in raw EEG data and giving out movement commands to the wheelchair. A MATLAB program was developed that communicates with the ThinkGear module to receive raw EEG waves. The program sends an instruction as a single byte to the Arduino in a wheelchair. This research work utilized GUIDE, which is an in-built GUI development tool of MATLAB. Using GUIDE, a simple GUI window was developed which displays the graph of the incoming raw brain wave from the TGAM. The GUI iterates through the directions while displaying images of arrows, making it easier for the patient to choose his next movement. The patient must make a voluntary eye blink to choose the desired direction. Once a direction is chosen, the program connects with the HC-05 Bluetooth module in the wheelchair and sends a character denoting the choice of the user. The program also plays a sound through the computer ${ }^{\text {ee }}$ speakers when an eye blink is detected.

The wheelchair chassis in this research is a miniature model and thus powered by two DC motors. Since the wheelchair must be able to move bidirectionally, a dual H-Bridge L298N motor driver module was used. One H-Bridge controlling each motor. The input to the L298N motor driver comes from an Arduino UNO [9] which is pre-coded to turn byte inputs from the computer to motor commands. An HC-05 Bluetooth module on the chassis connects to the computer and takes in a single-byte character. This character is decoded by the Arduino code to determine the direction of movement.

\subsection{GUI Development}

Previous works on wheelchair control based on eye blink patterns have been limited to very few control elements due to the limited number of patterns that could be created with eye blinks. For example, in [12], every time the patient blinks their eyes twice rapidly, the wheelchair will turn on or off. This accounted for bidirectional control of the wheelchair but in a real case scenario, four or more control patterns are required. This problem could be solved by using a GUI to increase the control options while still taking simple eye blinks as input from the user. This also avoids the need for the user to make complex eye blink patterns to navigate their wheelchair.

The direction of movement of the wheelchair is controlled by a program that is written in MATLAB software. This program determines the user"s choice and the corresponding movement of motors in the wheelchair. The user is given the choice to select one of the four directions: forward, backward, right, or left. To make this well-timed choice, it is important to make a clean and simple GUI for the user to interact with the computer. Graphical user interfaces usually provide point-to-click control of a software, relieving the need for the user to type commands [22]. However, the purpose of a GUI in this work is to provide the user with a visual representation of four choices to select from, aiding them to make timely decisions via eye blinks. Graphical User Interface Development Environment (GUIDE) is a development environment in MATLAB that provides us the tools to design Graphical User Interfaces and create custom applications. GUIDE has two primary tasks: To layout the visual components of a graphical user interface and to program app behavior.

It provides the user with the ability to create a GUI interactively. This means that the GUI windows, buttons, pop-up menus, etc. can be created just by dragging and dropping the components. Once the user interface is created, GUIDE automatically creates its own program in MATLAB with the necessary callback functions. These callback functions can then be edited to assign functionality to each component in the GUI and bring it to life. The initial stage of the GUI takes a few seconds to connect to the HC-05 Bluetooth module on the wheelchair. All the assets are loaded during this stage. In the following stage shown in Figure. 7(a), MATLAB has successfully established a Bluetooth connection between the computer and the HC-05 in the wheelchair. The GUI prompts the user to press the „Start ${ }^{e c}$ button to proceed. It can be noticed that the status is empty and EEG waveforms have not yet been graphed since the computer has not yet connected to the TGAM. 


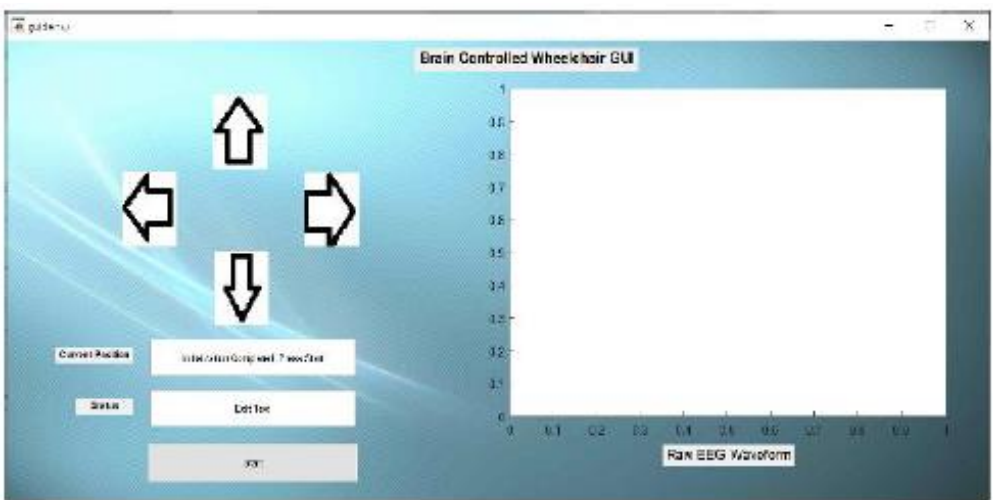

(a)

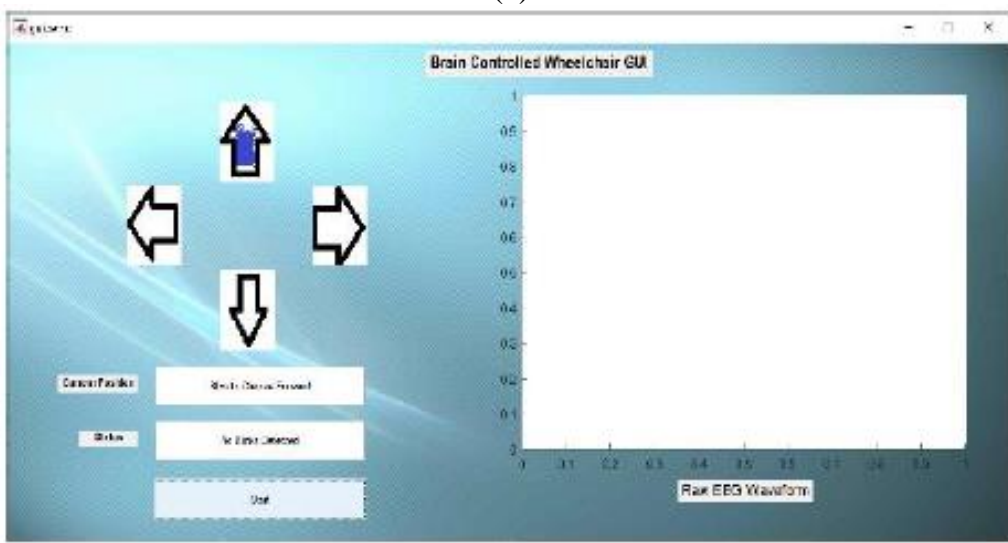

(b)

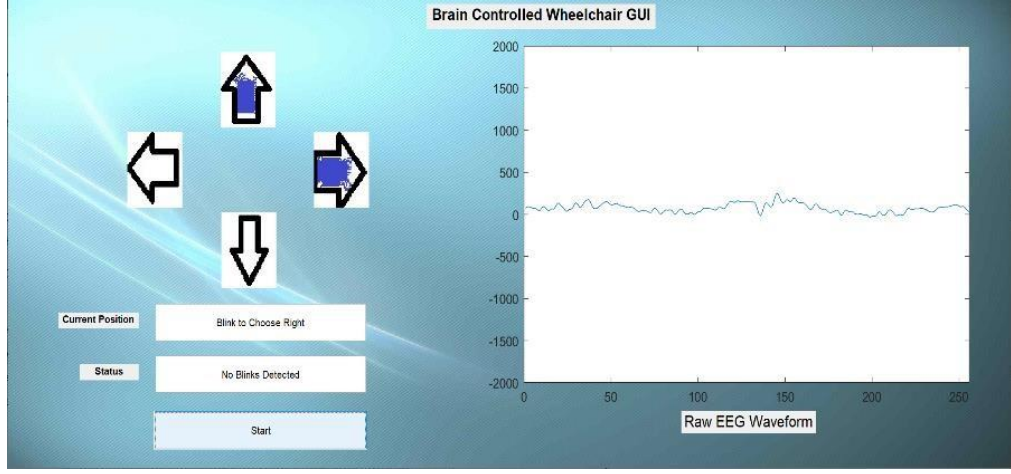

(c)

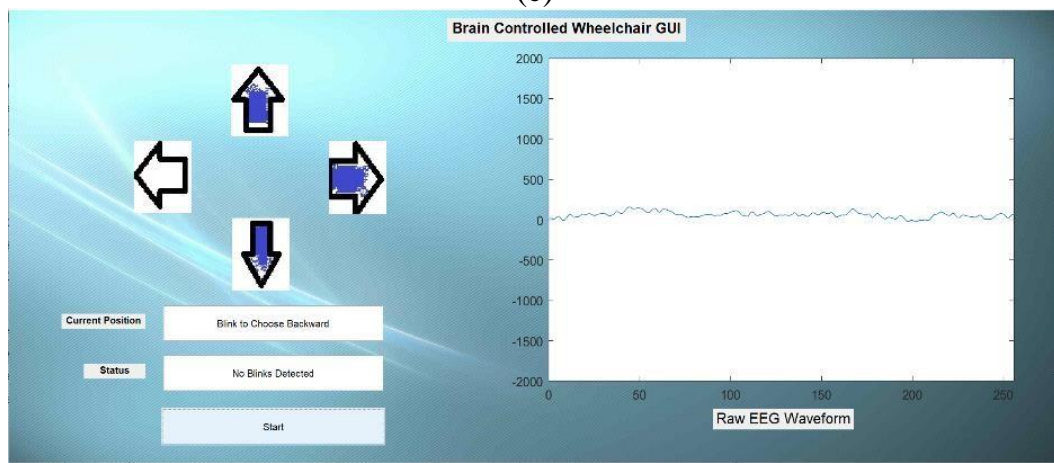

(d)

Figure 7. (a) GUI ready and waiting for the user to press 'Start' (b) GUI offering the user to select the forward movement (c) GUI offering the user to select right turn (d) GUI offering the user to select the reverse movement

The next stage shown in Figure 7(b) indicates that the user has pressed „Start ${ }^{e e}$ and MATLAB has successfully established a connection with ThinkGear. Data packets are plotted on the right, which gives the 
real-time updated EEG waveform. The navigation arrow „Forward ${ }^{e e}$ is highlighted, indicating that an eye blink could command the wheelchair to move forward. The user gets 3 seconds for selecting a direction. If a direction is not selected, the GUI automatically offers the next direction in a clockwise direction.

\subsection{Blink Strength Threshold}

Using eye blinks as the control element in this work came with a practical hurdle. Blinking is a bodily function that we do involuntarily, which lubricates and cleans our eyes by momentarily spreading tears over the surface of the eyes. It would not be feasible if every eye blink of the patient is registered as a movement command for the wheelchair. A person would naturally blink their eyes while sitting on the wheelchair and if all those blinks are detected by the MATLAB program as a command, the wheelchair would make unnecessary movements. Therefore, it was important to distinguish a natural eye blink from a command eye blink.

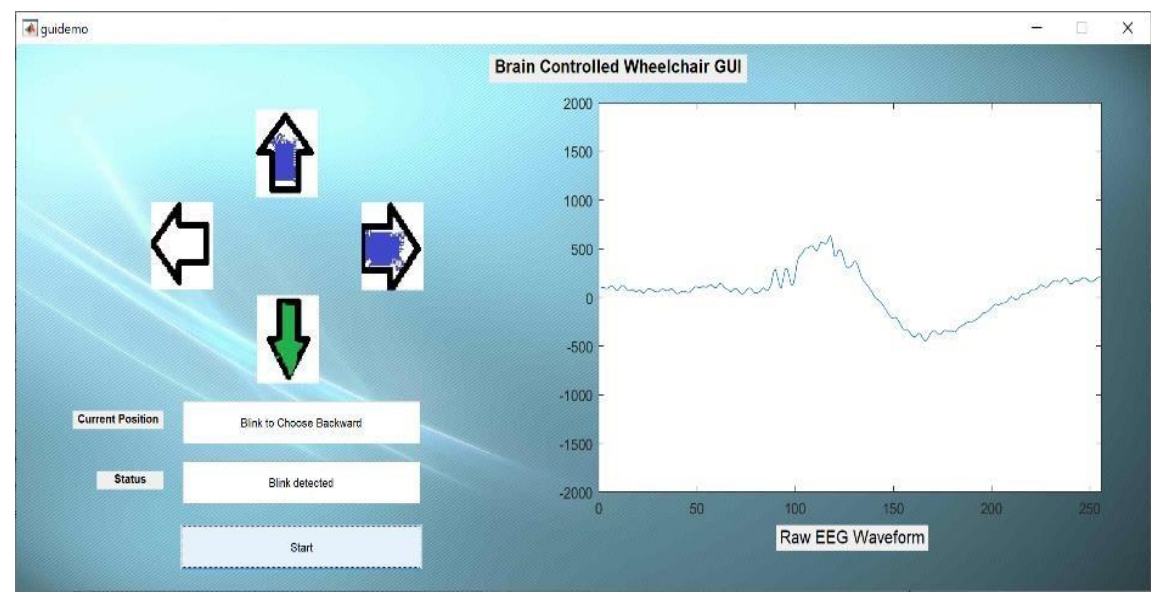

Figure 7(e). GUI showing the selection of reverse movement

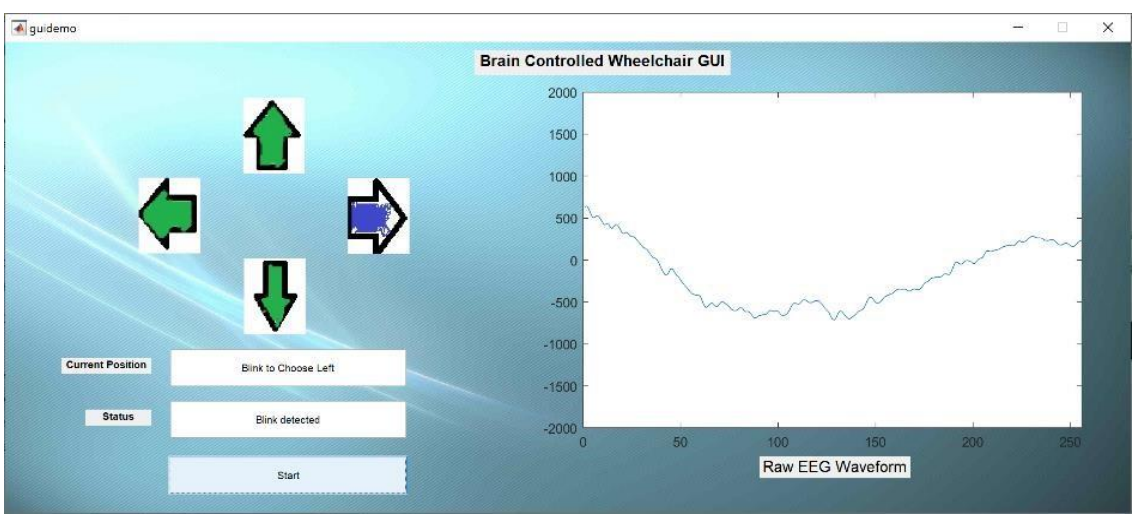

Figure 7(f). GUI showing multiple selections

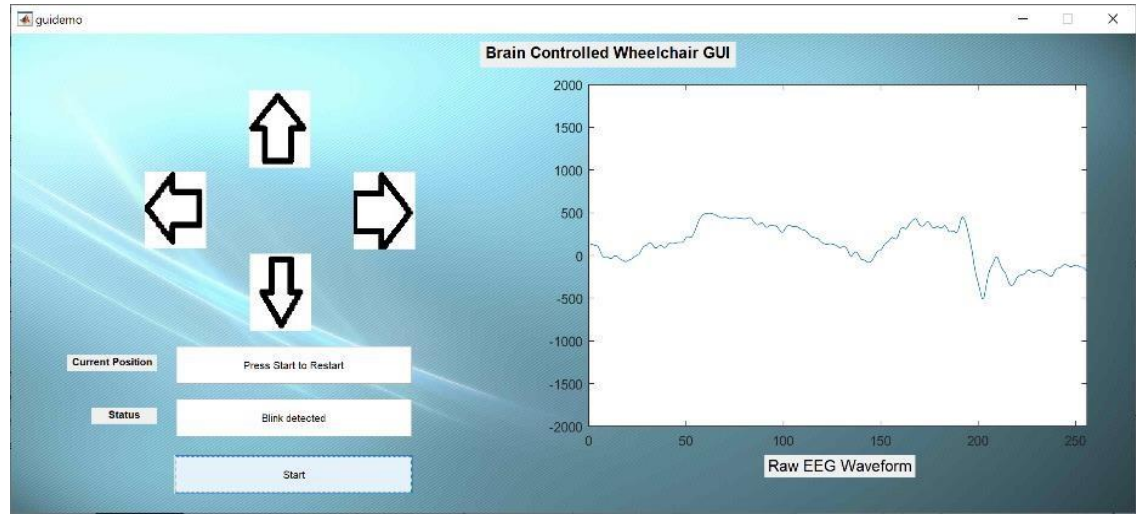

Figure $7(\mathrm{~g})$. GUI prompting the user to press 'Restart' 


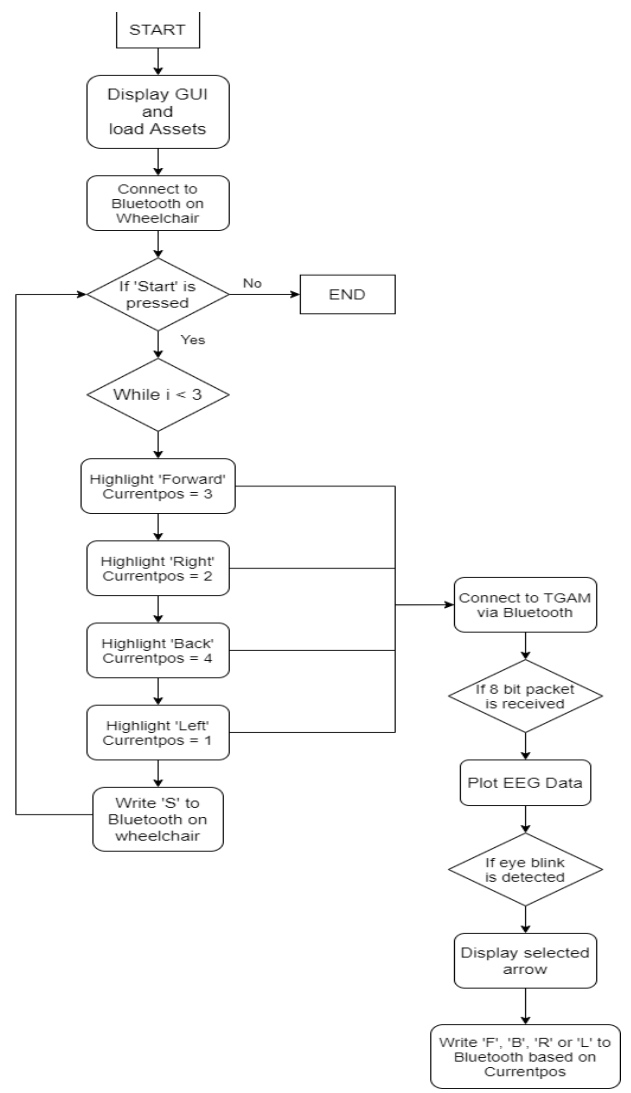

Figure 8. Flowchart of the MATLAB program

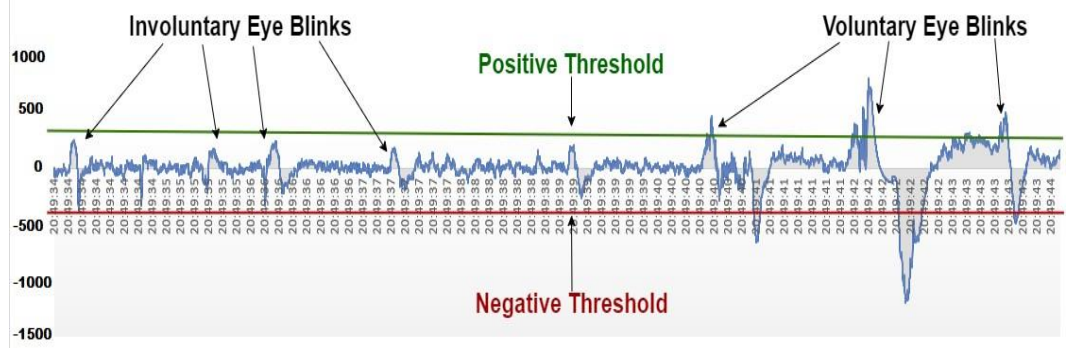

Figure. 9. EEG waveforms indicating the positive and negative thresholds separate voluntary eye blinks from involuntary eye blinks

To make this distinction, a positive and negative blink strength threshold is used. The positive blink strength threshold is the largest value of incoming EEG data bit which is classified as a natural eye blink in the MATLAB program. Whereas, negative blink strength threshold is the smallest value of incoming EEG data bit which is classified as a natural eye blink. Any EEG signal above the positive threshold or below the negative threshold is classified as a forced eye blink, which would be taken as a command for the wheelchair. This value was set after testing the headwear on five test subjects. The test subjects are not informed of the process being monitored while taking samples of natural eye blinks to make sure that they are not conscious of the blinking process. Later, they are asked to make the subtlest forced eye blinks. The positive and negative peak values of these test samples are analyzed to find out the average values that precisely separated EEG data collected from natural eye blinks from the EEG data collected from forced eye blinks. These average values are fixed as the positive and negative blink strength thresholds as shown in Figure.9. The positive threshold is indicated with a green line and it can be observed that the positive peaks of the voluntary eye blinks exceed this line. The negative threshold is indicated with a red line and the negative peaks of the voluntary eye blinks exceed this line.

\subsection{Parallax Data Acquisition}

Parallax Data Acquisition tool is a software add-on for Microsoft Excel, which allows the user to acquire data from micro-controllers. The data that is exported by the micro-controllers are taken real-time and 
entered into the columns of an Excel Spreadsheet. PLX-DAQ is often used in sensor monitoring because of its real-time display, plotting, and analyzing capabilities. PLX-DAQ can also add its own time stamps of the data arrival and save it with the data. To save and analyze the EEG data that is acquired by the headwear, we use PLX-DAQ as mentioned in [23]. PLX-DAQ constantly looks for data communication through the serial ports at a specified baud rate. Once the connection is established, it logs data onto the columns of a Microsoft Excel spreadsheet. However, the data must be exported in a comma-separated format from the Arduino for the plugin to be able to read it. The baud rate of Arduino output should be the same as the baud rate set on the PLX-DAQ control panel.

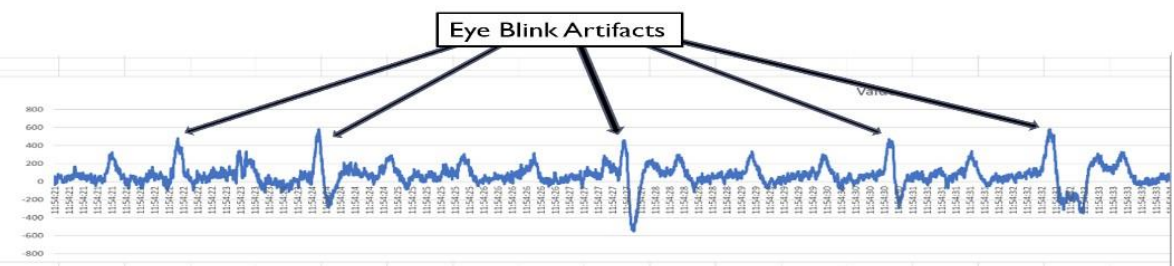

Figure 10. 2-D line plot of EEG data logged through PLX-DAQ, showing the eye blink artifacts

The incoming EEG data can be analyzed using Excel charts. Figure. 10 shows an example of an EEG signal plotted using a 2-D line chart. Several eye blink artifacts can be observed from the plot.

\subsection{H-Bridge for motor control}

An H-bridge is an electrical circuit that allows a voltage difference to be applied across a load in either direction. In our work, an H-Bridge is used for direction control of motors which enables forward and backward movement of the wheelchair. The H-Bridge consists of four switching elements, mostly bi-polar or Field Effect Transistors (FET), with the motor at the center in an H-shaped configuration as shown in Figure. 11. The diodes used are usually Schottky. The top of the H-bridge is connected to a power supply, and the bottom is grounded.
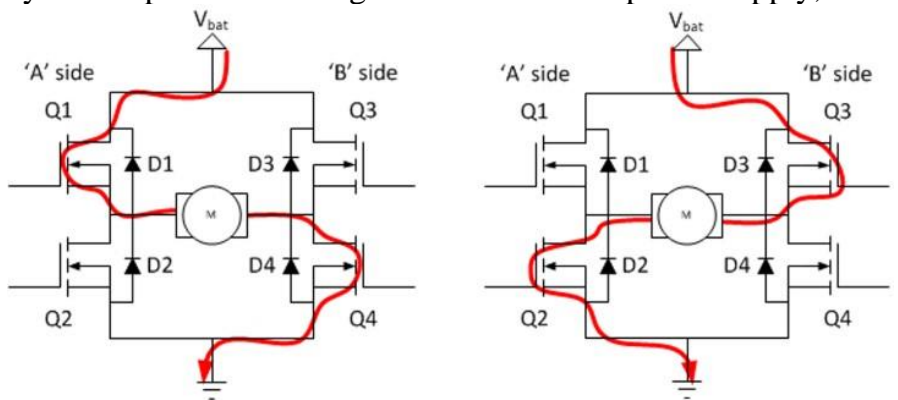

Figure 11. Illustration of current flow for a) Forward rotation of Motor and b) Backward rotation of Motor

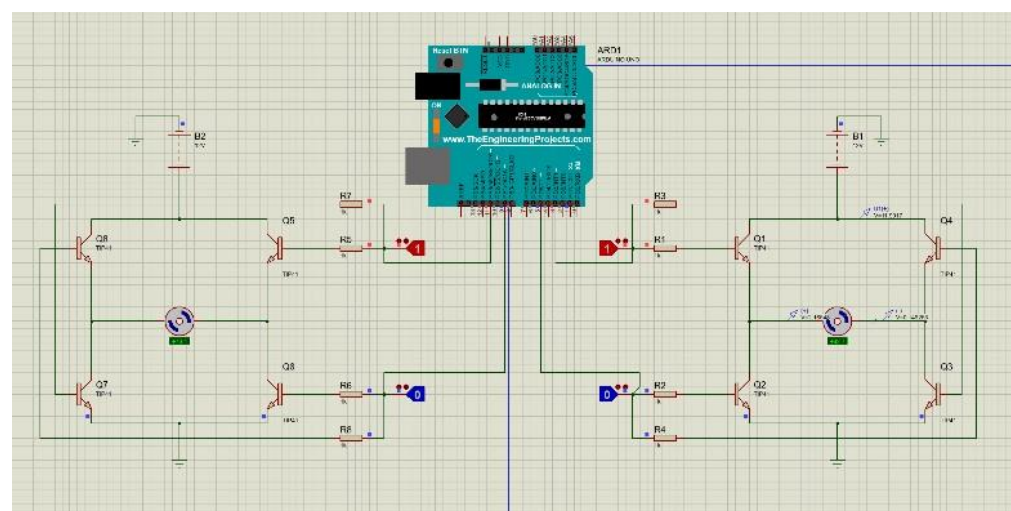

Figure 12. Simulation of dual H-Bridge motor driver in Proteus Design Suite

The basic operation of an H-Bridge [24] is as follows. When the switches Q1 and Q4 are turned on, the current conducts through Vbat - Q1 - Q4 - Gnd. This makes the motor turn in one direction. When the switches Q3 and Q2 are turned on, the current conducts through Vbat - Q3 - Q2 - Gnd, which makes the shaft of the motor rotate in the opposite direction. 


\section{HARDWARE IMPLEMENTATION}

Table 1. Design Specifications

\begin{tabular}{|c|c|c|c|}
\hline COMPONENT & SPECIFICATION & COMPONENT & SPECIFICATION \\
\hline $\begin{array}{l}\text { Think Gear } \\
\text { ASIC module }\end{array}$ & $\begin{array}{l}\text { Operating voltage }-2.97 \mathrm{~V} \text { to } 3.63 \mathrm{~V} \\
\mathrm{UART} \text { output baud rate }-1200,9600 \text {, } \\
57600 \\
\text { Sampling frequency }-512 \text { bits per second } \\
\text { Max power consumption }-15 \mathrm{~mA} @ 3.3 \\
\text { V } \\
\text { Quantity }-1 \text {, Weight }-130 \mathrm{mg}\end{array}$ & DC Battery & $\begin{array}{l}\text { Type - AAA } \\
\text { Voltage }-1.5 \mathrm{~V} \\
\text { Quantity }-3\end{array}$ \\
\hline $\begin{array}{l}\text { HC-05 } \\
\text { Bluetooth module }\end{array}$ & $\begin{array}{l}\text { Operating voltage }-5 \mathrm{~V} \\
\text { Operating current }-30 \mathrm{~mA} \\
\text { Communication range }- \text { less than } 100 \mathrm{~m} \\
\text { Supported baud rates }-9600,57600 \\
\text { Quantity }-2 \\
\text { Weight }-9.07 \mathrm{gm}\end{array}$ & $\begin{array}{l}\text { DC Plug } \\
\text { Adapter }\end{array}$ & $\begin{array}{l}\text { Input Voltage }(\mathrm{V})-100 \sim \\
280 \text { VAC @ } 50 \sim 60 \mathrm{~Hz} \\
\text { Input current }-100 \mathrm{~mA} \\
\text { Output Power }-9 \mathrm{~V}, 2 \text { A Input } \\
\text { Plug - 2-Pin EU type. Output Plug } \\
-5.5 \mathrm{~mm} \text { DC plug. }\end{array}$ \\
\hline $\begin{array}{c}\text { EEG } \\
\text { Electrodes }\end{array}$ & $\begin{array}{l}\text { Diameter }-1 \mathrm{~cm} \\
\text { Type }- \text { Silver } \\
\text { Quantity }-1 \\
\text { Driver voltage }-5 \mathrm{~V} \text { to } 35 \mathrm{~V}\end{array}$ & DC Motor & $\begin{array}{l}\text { Operating Voltage }-12 \mathrm{~V} \\
\text { Speed }-60 \mathrm{RPM} \text { at } 12 \mathrm{~V} \\
\text { Rated Torque }-3.6 \mathrm{Kg}-\mathrm{cm} \\
\text { Stall Torque }-15 \mathrm{Kg}-\mathrm{cm} \\
\text { Quantity }-2\end{array}$ \\
\hline $\begin{array}{l}\text { L298N Dual H- } \\
\text { bridge } \\
\text { Motor driver }\end{array}$ & $\begin{array}{l}\text { Driver current }-2 \mathrm{~A} \\
\text { Max power }-25 \mathrm{~W} \\
\text { Operating current range }-0 \text { to } 36 \mathrm{~mA} \\
\text { Weight }-26 \mathrm{gm} \\
\text { Quantity }-1\end{array}$ & & \\
\hline
\end{tabular}

\subsection{Headwear}

To minimize external disturbances during signal acquisition, a specialized signal acquisition module that is intended for EEG applications is used. NeuroSky Technologies is one of the leading research groups that focus on the advancement of biosensors and provides various personalized healthcare and wellness metrics [25,26]. Their work on the development of EEG sensors has proven to bring high-grade consumer products to provide vast solutions. ThinkGear ASIC Module (TGAM) is Neurosky ${ }^{e}$ s frontline brain wave sensor used for various applications [21].

A TGAM module as shown in Figure. 13(b), reads the raw EEG waveforms and displays them on a computer $[23,25]$. The TGAM module is powered by a battery pack that supplies a cumulative voltage of $4.5 \mathrm{~V}$. A single channel non-invasive silver EEG electrode placed at the pre-frontal lobe (FP1) $[17,27,28]$ extracts electrical signals from the scalp of the subject. This particular FP1 placement is used because FP1 and FP2 placements show the greatest deflection of potential in a time-domain EEG waveform [29]. A reference electrode clipped to the left ear lobe of the subject provides ground to the circuit. Figure. 13(a) shows the electrodes on the headwear and how it is to be placed on the subjectes head.

\subsection{Wheelchair Chassis}

The wheelchair chassis used here is a down-sized model of a wheelchair giving less emphasis to the mechanical aspects. The MATLAB software sends a single byte command to the wheelchair chassis to make a movement. This command is received by an HC-05 Bluetooth module mounted on the wheelchair chassis. This character byte is then sent to an Arduino UNO [13] which then decodes the character to its corresponding

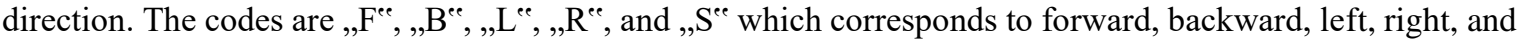
stop.

To provide bidirectional control of the motors, the output from the Arduino is given to an L298N motor driver and not directly to the DC motors. L298N has two inputs for each of the two motors. These are the terminals of the H-Bridges and the direction of rotation of a motor shaft depends on which pin is HIGH and which pin is LOW. The truth table for the direction of movement of the wheelchair is given below. 

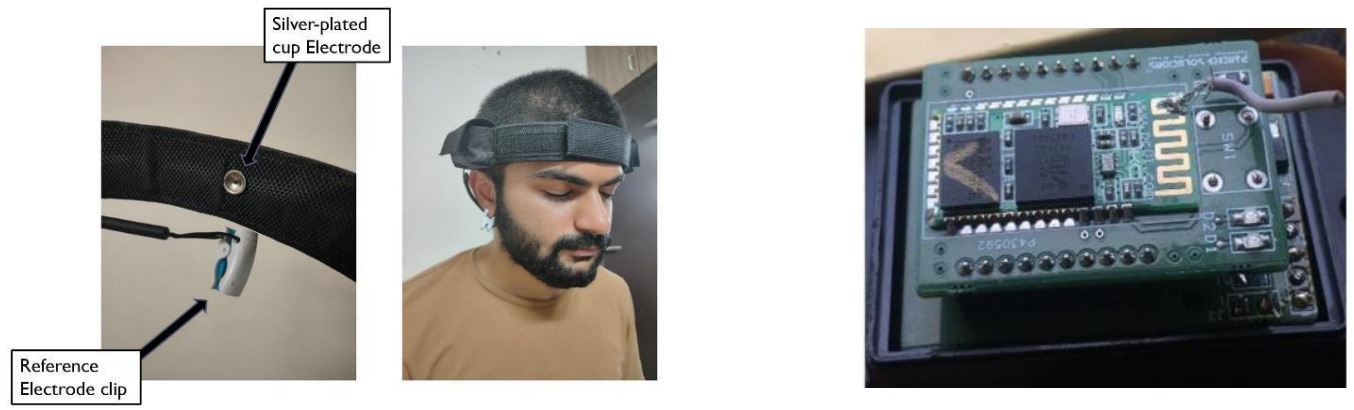

Figure. 13(a). Demonstration of the electrodes of the headwear and its proper placement on the head (b) ThinkGear ASIC Module integrated with a Bluetooth module

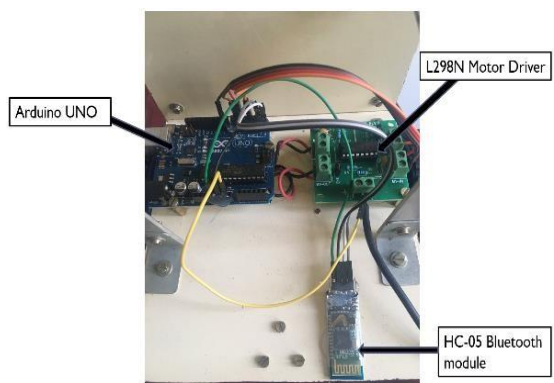

Figure. 14(a). Components in the wheelchair chassis

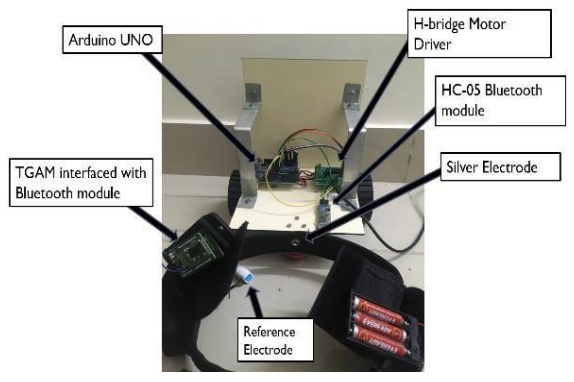

Figure. 14(b). Components of the wheelchair chassis and the headwear

Table 2. The truth table for wheelchair movements

\begin{tabular}{llllc}
\hline $\begin{array}{c}\text { Right } \\
\text { Motor A }\end{array}$ & $\begin{array}{c}\text { Left Motor } \\
\text { B }\end{array}$ & $\begin{array}{c}\text { Left Motor } \\
\text { A }\end{array}$ & $\begin{array}{c}\text { Wheelchair } \\
\text { Motor B }\end{array}$ & Movement \\
\hline High & Low & High & Low & Forward \\
Low & High & Low & High & Reverse \\
Low & High & High & Low & Right \\
High & Low & Low & High & Left \\
Low & Low & Low & Low & Stop \\
\hline
\end{tabular}

Figure 14(b) shows the components of the headwear along with the wheelchair chassis. The wheelchair works by interconnecting three separate modules, namely, the EEG signal acquisition headwear, a computer, and the wheelchair chassis.

\section{TEST RESULTS}

The human brain is unique for each and every person and it was important to study the performance of the prototype on different individuals. The brainwaves differ from person to person as well as from the

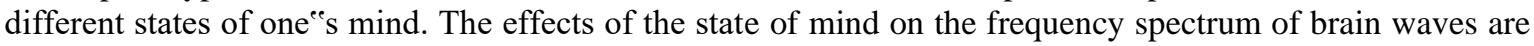
discussed in section II. A. To study the effect of the subjectes state of mind and the variation of EEG signals from person to person, real-time testing is carried out. The work tested on three test subjects who are in their early adulthood. Before testing, they are asked to describe their present state of mind and their emotions are noted down. Interestingly, their brain waves corresponded to their calmness, attention, and state of mind during testing. Figures 15(b), 16(b), and 17(b) show the EEG waveforms as time-domain signals.

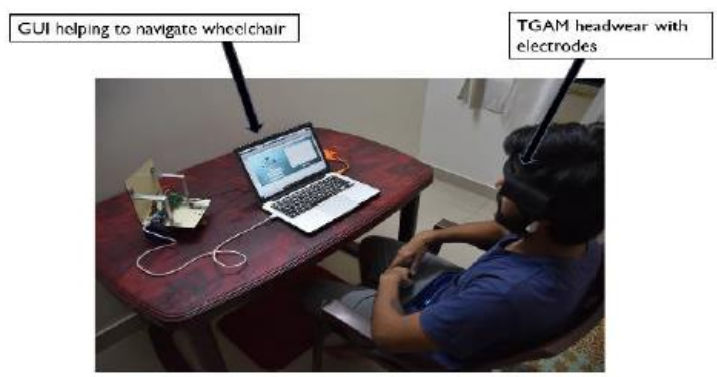

Figure 15. (a) Test subject-1 


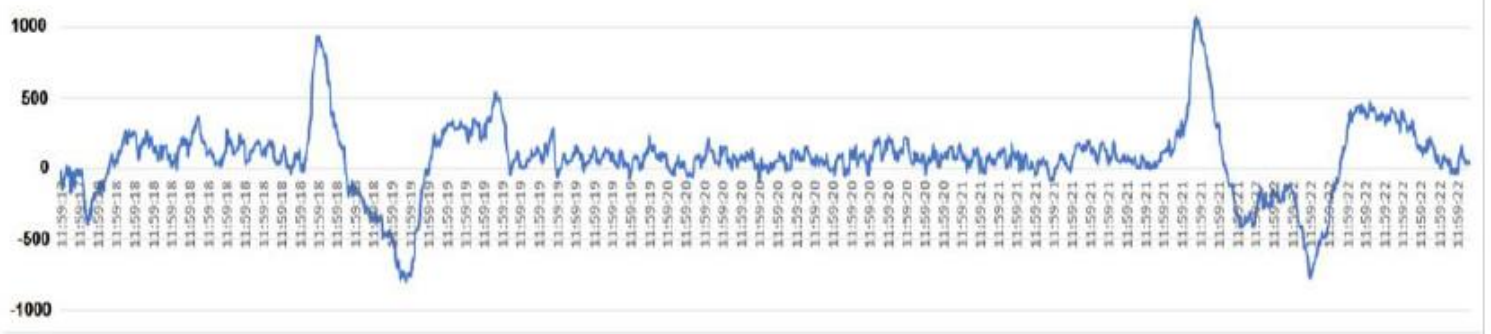

Figure 15. (b) EEG waveform of Test subject-1

The test setup of the first Test subject-1 is shown in Figure. 15(a). The subject is wearing the headwear making sure that the frontal EEG electrode is placed precisely at FP1 [17, 27]. Before electrode placement, the skin at FP1 is cleaned with cotton to reduce skin impedance. Without scalp preparation, the EEG waves are too distorted to obtain any valuable data. The ground electrode is clipped to the subject"s left ear lobe. The subject is then seated in front of a computer with the GUI that helps him to navigate the wheelchair. The subject can also view his own brain waves in real-time on the GUI. The subject is advised to keep his body relaxed and to avoid sudden movements, so as to minimize the noise in EEG signals.

The waveform in Figure. 15(b) shows the EEG signals of Test subject-1 plotted against time on the $\mathrm{X}$-axis. During preparation, the subject informed that he was happy, calm, and relaxed. It can be observed that his EEG waves are relatively clean without much disturbances other than the eye blink artifacts. Alpha and Theta waves (4 to $12 \mathrm{~Hz}$ ) dominate the brain waves when the subject is deeply relaxed. Another peculiar aspect of his EEG signal is the distinct style of eye blink artifact. They are less steep and more stepped than that of the other two test subjects. Absence of noise accounts to the focused state of his mind.

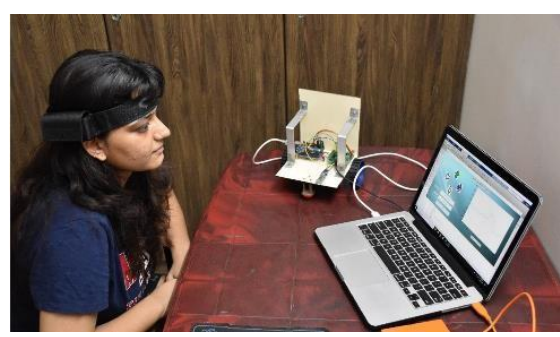

Figure 16. (a) Test subject-2

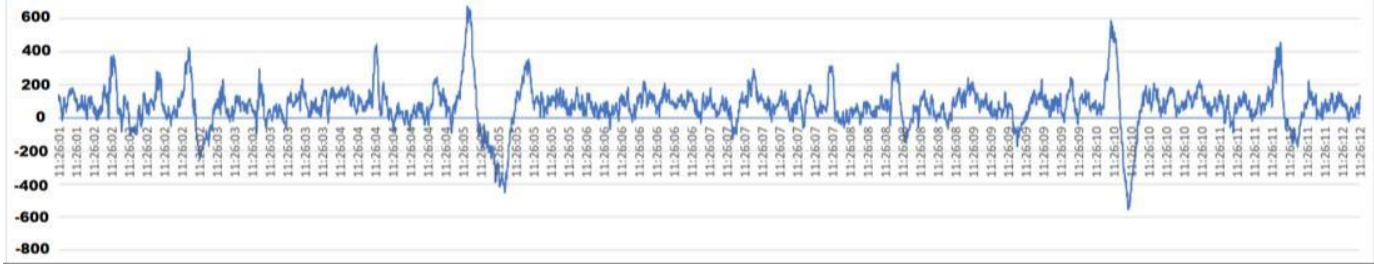

Figure 16. (b) EEG waveform of Test subject-2

Second (Test subject-2), described her state of mind as anxious and a little stressed due to work. Anxiety increases the Beta frequency band $(12-35 \mathrm{~Hz})$ waves in the EEG signal. Her EEG waves in Figure. 16(b) show a few disturbances which do not correspond to eye blinks. The source of these artifacts is unknown. The eye blink artifacts are steep and sudden as compared to the stepped and cleaner artifacts of Mr. Vishwa Prasad as shown in Figure 15(b).

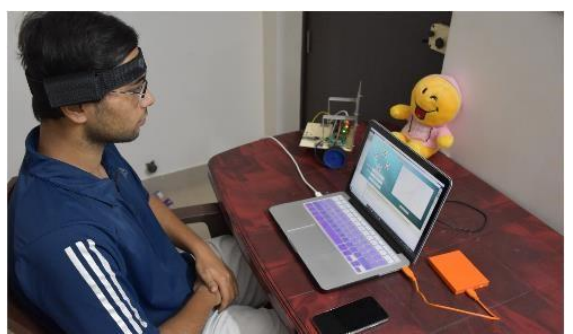

Figure 17. (a) Test subject-3 


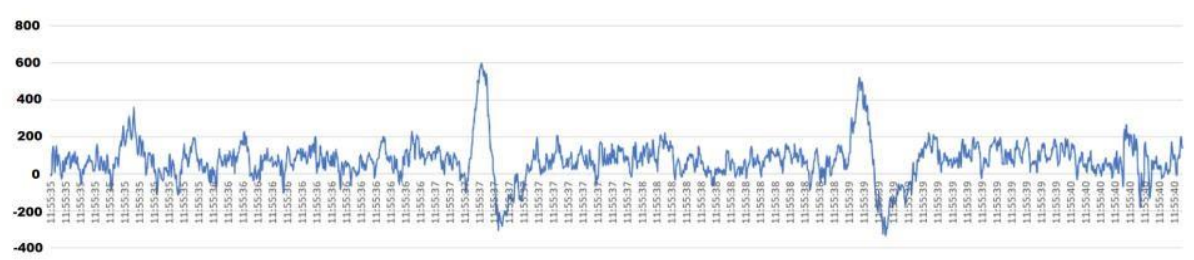

Figure 17. (b) EEG waveform of Test subject-3

The third (Test subject-3) indicated that he felt sleepy. The EEG signals as shown in Figure. 17(b) are dominated by Delta waves $(0.5$ to $4 \mathrm{~Hz})$ when the subject is in a sleepy mood. It can be observed that the signal has less noise as compared to the subject-2 signal. However, the eye blink artifacts are distinguishably visible and steep.

A few inferences can be made from the real-time testing on the above three test subjects

a. The mental state of the subject shows a direct correlation to their EEG waves.

b. The more relaxed the person is, the easier it is to distinguish eye blink artifacts from their brain waves.

c. Proper scalp preparation is important to minimize the noise in the EEG waves.

Table 3. Observations from real-time testing on test subjects

\begin{tabular}{lllll}
\hline Subject & Age & Mental State & Wave frequency & EEG Wave Observation \\
\hline Test subject-1 & 22 & Happy, Relaxed & 4 to $12 \mathrm{~Hz}$ & $\begin{array}{l}\text { Clean, Distinguishable and Stepped eye } \\
\text { blink artifacts }\end{array}$ \\
Test subject-2 & 21 & Stressed, Anxious & 12 to $35 \mathrm{~Hz}$ & $\begin{array}{l}\text { Unaccounted distortions and subtle eye } \\
\text { blink artifacts } \\
\text { Test subject-3 }\end{array}$ \\
22 & Sleepy, Relaxed & 0.5 to $4 \mathrm{~Hz}$ & $\begin{array}{l}\text { Steep and distinguishable eye blink } \\
\text { artifacts with noisy waves }\end{array}$ \\
\hline
\end{tabular}

Along with the analysis of EEG data obtained from real-time testing, the miniature wheelchair was also tested to find out how efficiently it functions. There are time lags between the command from the subject and the wheelchaires response to it. This is because MATLAB takes some time to process and send the commands to the wheelchair. The response time of the wheelchair would vary according to the processing power of the computer used. The time complexity of the MATLAB algorithm also plays a major role in adding to this delay. Table 4 shows the average results after 10 test runs of the wheelchair.

Table 4. Average results of wheelchair testing

\begin{tabular}{ll}
\hline \multicolumn{1}{c}{ Attribute } & Results \\
\hline Time for which the wheelchair moves for each eye blink & 2 seconds \\
Minimum time between two consecutive blinks to be registered & 4 seconds \\
Response time of wheelchair after an eye blink & 0.5 seconds \\
Wheel rotations per minute & $60 \mathrm{rpm}$ \\
Speed of the wheelchair & $0.79 \mathrm{~km} / \mathrm{hr}$ \\
Average turn time & 2 seconds \\
Time given to the user to make an eye blink for selecting a direction & 3 seconds \\
\hline
\end{tabular}

The accuracy of the BCI wheelchair is a very important measure as it determines the safety and the effectiveness of the wheelchair for its users. An experiment was carried out on five individuals of ages ranging from 23 to 87. Each individual was asked to make a random wheelchair movement through eye blinks. Each attempt was marked as either successful or unsuccessful. A successful attempt is counted when an eye blink is registered, correctly classified as the intended command and the command is executed by the wheelchair. Fifty such trials were carried out on each of the individuals and the results are shown in Table 5. The average command detection and execution accuracy was calculated as 96.4\%. A previously developed BCI system that utilizes EOG technology has shown a command detection and execution accuracy of 93.89\% [10]. This edge in the accuracy could be attributed to the higher Signal-to-Noise Ratio (SNR) of the BCI system or/and owing to the better accuracy of EEG technology for this application. The quick response time of the welloptimized MATLAB program could also have contributed to the user selection accuracy. Although the BCI system described in this paper requires an additional screen in front of the user to display the graphical user interface, its high accuracy can also be attributed to the presence of a user-friendly GUI. Figure 18 shows a line plot indicating the correlation between the test subjectes age and the corresponding accuracy of the 
wheelchair. A downward trend can be observed in the accuracy as the age of the subject increases. This is observed to be due to two reasons. The older the subject is, the weaker their voluntary eye blinks are, and therefore, the chances of their voluntary eye blinks to be classified as a control command decreases. Secondly, older individuals struggled to keep up with the pace of the GUI and failed to make timely voluntary eye blinks.

Table 5. Wheelchair performance on five test subjects for calculating average command detection and

\begin{tabular}{ccccc}
\hline Subject & Age & $\begin{array}{c}\text { Total } \\
\text { attempts }\end{array}$ & $\begin{array}{c}\text { Successful } \\
\text { attempts }\end{array}$ & $\begin{array}{c}\text { Command detection and } \\
\text { execution accuracy }\end{array}$ \\
\hline Test subject-1 & 23 & 50 & 50 & $100 \%$ \\
Test subject-2 & 41 & 50 & 49 & $98 \%$ \\
Test subject-3 & 59 & 50 & 48 & $98 \%$ \\
Test subject-4 & 66 & 50 & 49 & $96 \%$ \\
Test subject-5 & 87 & 50 & 45 & $90 \%$ \\
\hline
\end{tabular}

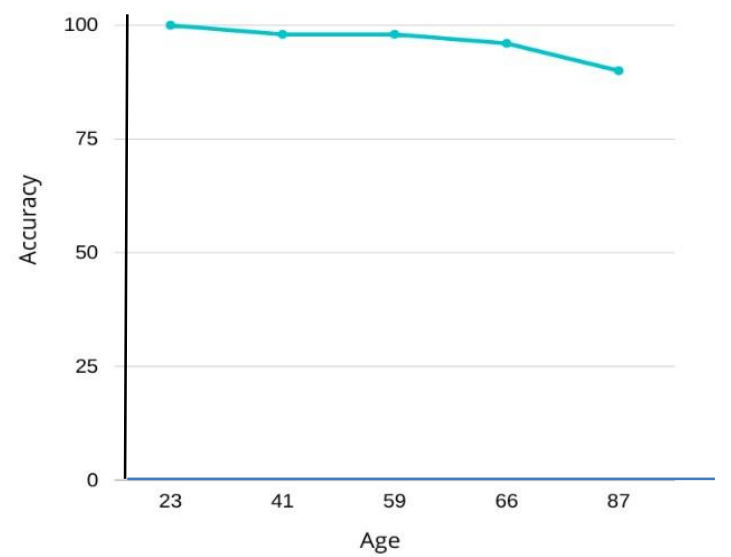

Figure 18. Correlation of the wheelchaires accuracy with the age of the subject

BCI research that has been done in the past years, on decoding the clockwork inside the human brain has brought major technological advancements resulting in better solutions for improving the lives of many physically challenged people. This research sheds light on the possibilities of how to utilize human brain wave signals to distinguish the eye blinks and use them as commands. To develop the prototype in this research for real-life application, a human-size wheelchair can be designed with powerful motors to carry paralyzed patients, utilizing the same underlying technology used in this prototype. If a component of this product becomes faulty, it can be easily replaced or repaired since it is modular in nature. The security of the product is ensured by password-protected Bluetooth connections. The components of the work are handpicked carefully to make the product cost-effective. This prototype can be developed into a product and introduced to the market at an estimated cost of \$210. Therefore, upscaling this work would be profitable and viable for industrial production. This technology can also be developed to bring life to the daily activities of paralyzed individuals such as controlling home appliances [18, 21], playing video games, and interactive toys. It also has the potential to increase the work opportunities for the disabled, thus enhancing the quality of their life.

\section{CONCLUSION}

This work commenced as a research-based work to understand human brain waves and how various artifacts could be captured from EEG signals for medical applications. Recognizing the struggle of severely paralyzed patients having to depend on others for their daily needs, the goal was set to provide a solution for their plight. Thus, a suitable wheelchair for the paralyzed patients that can be controlled solely by eye blinks is designed. The idea of choosing eye blink artifacts as the control element concluded from the variations in EEG patterns associated with various human stimuli. Since this research adapts eye blinks as the command, a wheelchair developed from this prototype can provide a helping hand to a wide spectrum of paralyzed patients across the world. Even the most paralyzed patients are capable of making eye blinks despite their disabilities. EEG signal acquisition with better signal filtering hardware revealed how these signals can control a device wirelessly without any physical gestures except for eye blinks. 


\section{REFERENCES}

[1] Smith CU. Cardiocentric neurophysiology: the persistence of a delusion. J Hist Neurosci. 2013;22(1):6-13. doi:10.1080/0964704X.2011.65089

[2] J. Van der Spiegel, M. Zhang and X. Liu, "The next-generation brain machine interface system for neuroscience research and neuroprosthetics development," 2017 IEEE 12th International Conference on ASIC (ASICON), Guiyang, 2017, pp. 436-439, doi: 10.1109/ASICON.2017.8252507.

[3] Sasikumar Gurumurthy, Vudi Sai Mahit and Ritwika Ghosh, "Analysis and simulation of brain signal data by EEG signal processing technique using MATLAB” et.al / International Journal of Engineering and Technology (IJET), Vol 5 No 3 Jun-Jul 2013.

[4] L. Rahman - et al., "A low cost Human Computer Interface for Disabled People based on Eye Blink detection using Brain Signal," TENCON 2019 - 2019 IEEE Region 10 Conference (TENCON), Kochi, India, 2019, pp. 19371941, doi:10.1109/TENCON.2019.8929452.

[5] Subha, D.P., Joseph, P.K., Acharya U, R. et al. EEG Signal Analysis: A Survey. J Med Syst 34, 195-212 (2010).

[6] Zhang J, Wang B, Zhang C, Xiao Y and Wang MY (2019) An EEG/EMG/EOG-Based Multimodal HumanMachine Interface to Real-Time Control of a Soft Robot Hand. Front. Neurorobot. 13:7. doi: 10.3389/fnbot.2019.00007

[7] Chevli, Daizy \& Kothiya, Animesh \& Patel, Jignal \& Purohit, Kavil \& Khambhati, Dimpal. (2019). Designing of Wheelchair to provide Ability to Move around for Disabled People using Electrooculography. International Journal of Engineering Trends and Technology. 67. 75-79. 10.14445/22315381/IJETT-V67I3P214.

[8] Gips, J., DiMattia, P., Curran, F., Olivieri, P.: Using EagleEyes-an electrodes based device for controlling the computer with your eyes to help people with special needs. In: Proceedings of the 5thInternational Conference on Computers Helping People with Special Needs, vol. 1, pp. 77-83 (1996).

[9] Thoumies, P., Charlier, J.R., Alecki, M., d eerceville, D., Heurtin,A., Mathe, J.F., Nadeau, G., Wiart, L.: Clinical and functional evaluation of a gaze controlled system for the severely handicapped. Spinal Cord. 36, 104-109 (1998).

[10] Choudhari et al. Biocybernetics and Biomedical Engineering 39, no. 3 (2019): 673-685.

[11] Nakanishi et al, "Wheelchair control system by using electrooculogram signal processing." In The 19th KoreaJapan Joint Workshop on Frontiers of Computer Vision, pp. 137-142. 2013.

[12] L. Rahman - et al., "A low cost Human Computer Interface for Disabled People based on Eye Blink detection using Brain Signal," TENCON 2019 - 2019 IEEE Region 10 Conference (TENCON), Kochi, India, 2019, pp. 19371941, doi:10.1109/TENCON.2019.8929452.

[13] I. A. Mirza et al., "Mind-controlled wheelchair using an EEG headset and arduino microcontroller," 2015 International Conference on Technologies for Sustainable Development (ICTSD), Mumbai, 2015, pp. 1-5, doi: 10.1109/ICTSD.2015.7095887.

[14] Królak, A., Strumiłło, P. Eye-blink detection system for human-computer interaction. Univ Access Inf Soc 11, 409_ 419 (2012). https://doi.org/10.1007/s10209-011-0256-6.

[15] Kucukyildiz, G., Ocak, H., Karakaya, S. et al. Design and Implementation of a Multi Sensor Based Brain Computer Interface for a Robotic Wheelchair. J Intell Robot Syst 87, 247-263 (2017).

[16] “Implementing a Low Noise Low Power Portable EEG Sensor System”, Jennifer Legaspi, Jordyn Rombola, Allison Thibault, Worcester Polytechnic Institute.

[17] J. A. I. R. Silva, F. E. Suarez Burgos and S. Wu, "Interactive Visualization of the Cranio-Cerebral Correspondences for 10/20, 10/10 and 10/5 Systems," 2016 29th SIBGRAPI Conference on Graphics, Patterns and Images (SIBGRAPI), Sao Paulo, 2016, pp. 424-431, doi: 10.1109/SIBGRAPI.2016.065.

[18] M. S. b Abd Rani and W. bt. Mansor, "Detection of eye blinks from EEG signals for home lighting system activation," 2009 6th International Symposium on Mechatronics and its Applications, Sharjah, 2009, pp. 1-4, doi: 10.1109/ISMA.2009.5164828

[19] Y. Xie and X. Li, "A brain-controlled wheelchair based on common spatial pattern," 2015 International Symposium on Bioelectronics and Bioinformatics (ISBB), Beijing, 2015, pp. 19-22, doi: 10.1109/ISBB.2015.7344913.

[20] Stamps K., Hamam Y. (2010) Towards Inexpensive BCI Control for Wheelchair Navigation in the Enabled Environment - A Hardware Survey. In: Yao Y., Sun R., Poggio T., Liu J., Zhong N., Huang J. (eds) Brain Informatics. BI 2010. Lecture Notes in Computer Science, vol 6334. Springer, Berlin, Heidelberg.

[21] D. Bright, A. Nair, D. Salvekar and S. Bhisikar, "EEG-based brain controlled prosthetic arm," 2016 Conference on Advances in Signal Processing (CASP), Pune, 2016, pp. 479-483, doi: 10.1109/CASP.2016.7746219.

[22] A. O. Selvı, A. Ferıkoğlu, D. Güzel and E. Karagöz, "Design and implementation of EEG signal based brain computer interface for eye blink detection," 2017 International Conference on Computer Science and Engineering (UBMK), Antalya, 2017, pp. 544-548, doi: 10.1109/UBMK.2017.8093458.

[23] M. A. H. Ali and N. A. Yusoff, "Development of Tele-Monitoring Attendance System Using RFID and PhotoCell," 2018 IEEE International Conference on Automatic Control and Intelligent Systems (I2CACIS), Shah Alam, 2018, pp. 83-88, doi: 10.1109/I2CACIS.2018.8603695. 
[24] V. Gupta, "Working and analysis of the H - bridge motor driver circuit designed for wheeled mobile robots," 2010 2nd International Conference on Advanced Computer Control, Shenyang, 2010, pp. 441-444, doi: 10.1109/ICACC.2010.5486818.

[25] A. Maksud, R. I. Chowdhury, T. T. Chowdhury, S. A. Fattah, C. Shahanaz and S. S. Chowdhury, "Low-cost EEG based electric wheelchair with advanced control features," TENCON 2017 - 2017 IEEE Region 10 Conference, Penang, 2017, pp. 2648-2653, doi: 10.1109/TENCON.2017.8228309.

[26] Sinha, U., \& Kanthi, M. (2016). Mind controlled wheelchair. International Journal of Control Theory and Applications, 9(39), 19-28.

[27] Awais, Muhammad Ahsan \& Yusoff, Mohd \& Yahya, Norashikin \& Ahmed, Zeeshan \& Qamar, Muhammad. (2020). Brain Controlled Wheelchair: A Smart Prototype. Journal of Physics Conference Series. 1529. 42075. 10.1088/1742-6596/1529/4/042075.

[28] Keerthi Kumar M, Chaitra Rai, Manisha R, Priyanka C B, Syeda Saniya Anis, 2019, EEG Controlled Smart Wheelchair for Disabled People, INTERNATIONAL JOURNAL OF ENGINEERING RESEARCH \& TECHNOLOGY (IJERT) NCRACES - 2019 (Volume 7, Issue 10).

[29] Babusiak, Branko \& Mohylová, Jitka. (2009). Eye-blink artifact detection in the EEG. 25/4. 10.1007/978-364203882-2_310.

\section{BIOGRAPHIES OF AUTHORS}

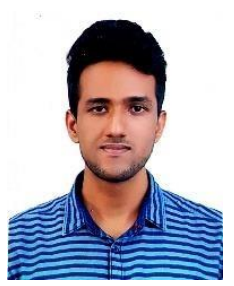

William C Francis received his B.Tech in Electrical and Electronics Engineering from

VIT University, Chennai. He is currently a graduate student at the University of Pennsylvania, USA. His research areas of interest include Biomedical instrumentation and Machine Learning.

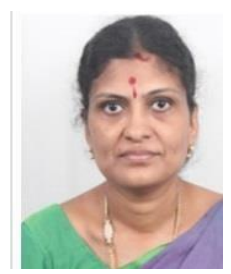

Umayal C. received her Bachelor of Engineering from University of Madras, Master of Engineering and Ph.D from Anna University. She is working as Associate professor in the School of Electrical Engineering, VIT, Chennai, India. She has authored many international and national level research papers on power factor correction in PMBLDC Drives.

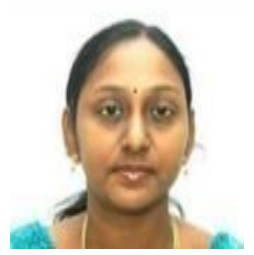

Kanimozhi G. received her Bachelor of Engineering from Bharathiyar University, Master of Engineering from Anna university and her Ph.D from VIT, Chennai. She is working as Assistant Professor (Selection Grade) in the School of Electrical Engineering, VIT Chennai. Her research area includes AC-DC converters for Electric vehicles, biomedical equipments, Electromagnetics, Multilevel inverters and DC-DC resonant converters. 